\title{
Modelagem e quantificação da incerteza espacial do potássio disponível no solo por simulações estocásticas
}

\author{
Ismênia Ribeiro de Oliveira(1), Daniel De Bortoli Teixeira(2), Alan Rodrigo Panosso ${ }^{(3)}$, \\ José Marques Júnior(2) e Gener Tadeu Pereira(2)
}

\begin{abstract}
(1)Universidade Federal do Maranhão, Centro de Ciências Agrárias e Ambientais, BR 222, Km 04, Boa Vista, CEP $65500-000$ Chapadinha MA, Brasil. E-mail: ismenia.ribeiro@ufma.br (2)Universidade Estadual Paulista (Unesp), Faculdade de Ciências Agrárias e Veterinárias, Via de Acesso Prof. Paulo Donato Castellane, s/no, CEP 14884-900 Jaboticabal, SP, Brasil. E-mail: daniel.dbt@hotmail.com, marques@fcav.unesp.br, genertp@fcav.unesp.br ${ }^{(3)}$ Unesp, Faculdade de Engenharia de llha Solteira, Avenida Brasil, no 56, CEP 15385-000 Ilha Solteira, SP, Brasil. E-mail: alanrp@mat.feis.unesp.br
\end{abstract}

Resumo - O objetivo deste trabalho foi avaliar o desempenho da simulação sequencial gaussiana (SSG) e da simulação sequencial indicatriz (SSI) na modelagem da incerteza das predições do K disponível em área de cana-de-açúcar, e comparar as simulações com o método já consagrado de krigagem ordinária (KO). Uma malha amostral com 626 pontos foi instalada em área de 200 ha, no Município de Tabapuã, em São Paulo. As simulações reproduziram a variabilidade dos dados amostrais de $\mathrm{K}$ disponível, enquanto a KO superestimou os baixos teores de $\mathrm{K}$ e subestimou os altos. O mapa de desvio-padrão obtido a partir da $\mathrm{KO}$ mostrou menor variação ao longo da área de estudo, quando comparado aos mapas obtidos a partir das simulações. A SSI obteve acurácia $22 \%$ superior à obtida pela SSG, na modelagem da função de distribuição condicional do K. As simulações apresentam maior eficiência que a KO para modelar incerteza na distribuição espacial do K. A SSI apresenta melhor desempenho que a SSG na estimativa dos teores de K disponível, em área de cana-de-açúcar.

Termos para indexação: fertilidade do solo, geoestatística, krigagem, simulação sequencial gaussiana, simulação sequencial indicatriz, variabilidade espacial.

\section{Modeling and quantification of available potassium spatial uncertainty in the soil by stochastic simulations}

\begin{abstract}
The objective of this work was to evaluate the performance of the sequential Gaussian simulation (SGS) and the sequential indicator simulation (SIS) for modeling the uncertainty of available K predictions in a sugarcane area, and to compare both simulations to the already established method of ordinary kriging (OK). A sampling grid with 626 points was installed in an area of 200 ha, in the municipality of Tabapuã, in the state of São Paulo, Brazil. The simulations reproduced the variability in the available K sample data, whereas OK overestimated the low K levels and underestimated the high ones. The standard deviation map obtained from OK showed less variation along the studied area when compared to the maps obtained from the simulations. SIS achieved an accuracy 22\% higher than that obtained by SGS for modeling the conditional distribution function of K. The simulations have higher efficiency than OK for modeling the uncertainty in the spatial distribution of K. SIS has better performance than SGS for estimating the levels of available K in sugarcane area.
\end{abstract}

Index terms: soil fertility, geostatistics, kriging, sequential Gaussian simulation, sequential indicator simulation, spatial variability.

\section{Introdução}

O Estado de São Paulo é o maior produtor de canade-açúcar (Saccharum officinarum L.), com 51,7\% da área total cultivada no Brasil, segundo dados da Companhia Nacional do Abastecimento (2014). A cultura consome aproximadamente $13 \%$ do total de fertilizantes utilizado anualmente no país (Otto et al., 2010), e o potássio é o nutriente mais absorvido.
Os solos tropicais brasileiros apresentam baixos teores de potássio na forma disponível (Benites et al., 2010), e a cana-de-açúcar responde bem à aplicação do nutriente. $\mathrm{O}$ estudo da variabilidade espacial de $\mathrm{K}$ é uma forma de prover informações que possibilitem o manejo adequado do nutriente e que reduzam os custos de produção, aumentem a produtividade das culturas e diminuam o impacto ambiental da fertilização potássica. 
A geoestatística é a principal ferramenta utilizada para a caracterização da variabilidade espacial. Os métodos tradicionais geoestatísticos, como a krigagem, não fornecem informações suficientes para quantificar as incertezas. Contudo, a avaliação da incerteza, associada aos métodos de predição dos atributos analisados, tem ganhado importância nos últimos anos (Bourennane et al., 2007; Goovaerts et al., 2008; Delbari et al., 2009; Zhao et al., 2009; Sun et al., 2012; Teixeira et al., 2012). Em geoestatística, a incerteza é uma medida indicativa da magnitude do erro de estimação dos atributos, uma vez que este não pode ser determinado com exatidão, pois, para isso, seria necessário o conhecimento do valor real do atributo em cada posição estimada (Isaaks \& Srisvastava, 1989). O conhecimento da incerteza é uma etapa importante no processo de avaliação da qualidade do interpolador utilizado e dos riscos envolvidos nos processos de tomada de decisão.

A krigagem ordinária $(\mathrm{KO})$ é o estimador mais utilizado em estudos sobre a variabilidade espacial de atributos químicos do solo (Sanchez et al., 2009; Souza et al., 2010; Camargo et al., 2013); porém, o método suaviza detalhes locais pela superestimação de valores baixos e pela subestimação de valores altos (Zhao et al., 2009). Além disso, a obtenção da variância da estimativa por esse método depende apenas da distribuição dos dados e das características do variograma ajustado, e não leva em consideração os valores dos dados, o que a torna uma medida incompleta da incerteza local (Soares, 2006).

Estudos recentes têm utilizado simulações estocásticas para avaliar e quantificar a incerteza das propriedades químicas do solo (Bourennane et al., 2010; Sun et al., 2012); entretanto, no Brasil, ainda são poucas as publicações nessa área (Silva Junior et al., 2012; Teixeira et al., 2012; Oliveira et al., 2013). A simulação sequencial gaussiana (SSG) e a simulação sequencial indicatriz (SSI) são os tipos mais comuns de simulação estocástica. A SSG é a mais utilizada em estudos sobre propriedades químicas do solo (Bourennane et al., 2007; Chai et al., 2007; Delbari et al., 2009; Zhao et al., 2009; Sun et al., 2012), enquanto a SSI (Delbari et al., 2010) é frequentemente utilizada na área ambiental (Juang et al., 2004; Zhao et al., 2005). Ao contrário da $\mathrm{KO}$, os modelos de simulação estocástica reproduzem a variabilidade espacial dos dados, o que elimina o efeito de suavização. Os algoritmos de simulação geram um conjunto de realizações e não uma "melhor estimativa", como na KO (Soares, 2006). Assim, esse conjunto de realizações pode ser utilizado para medir a incerteza das predições geradas pelos modelos probabilísticos.

O objetivo deste trabalho foi avaliar o desempenho da simulação sequencial gaussiana (SSG) e da simulação sequencial indicatriz (SSI) na modelagem da incerteza das predições do $\mathrm{K}$ disponível em área de cana-de-açúcar, e comparar as simulações com o método já consagrado de krigagem ordinária $(\mathrm{KO})$.

\section{Material e Métodos}

A área de estudo $\left(21^{\circ} 05^{\prime} 57,11^{\prime \prime S}, 4^{\circ} 01^{\prime} 02,08^{\prime \prime} \mathrm{W}\right)$ localiza-se no Município de Tabapuã, no noroeste do Estado de São Paulo. O clima da região, segundo a classificação de Thornthwaite, é do tipo megatérmico, subúmido chuvoso, com pequeno ou nenhum excedente de água e evapotranspiração de verão menor que $48 \%$ do total anual. O solo foi classificado como Argissolo Vermelho-Amarelo eutrófico de textura média/argilosa, de acordo o Sistema Brasileiro de Classificação de Solos (Santos et al., 2013). A área, de 200 ha, apresenta histórico de mais de 20 anos de cultivo de cana-deaçúcar com sistema de colheita queimada.

O solo foi amostrado nos pontos de cruzamento de uma malha com intervalos regulares de $50 \mathrm{~m}$, num total de 626 pontos. Desse total, cerca de $10 \%$ dos pontos amostrados (63) foram sorteados, antes do início da modelagem geoestatística, para compor o conjunto de dados utilizado na validação da modelagem (Bourennane et al., 2007); os 563 pontos restantes foram utilizados para as predições da variável nos locais não amostrados. As amostras foram coletadas nas profundidades de $0,00-0,20 \mathrm{~m}$, e o potássio disponível foi extraído pelo método da resina trocadora de íons, proposto por Raij et al. (2001). Esse banco de dados, juntamente com as metodologias de coleta, análise e classificação dos dados, é originário de pesquisas realizadas por Sanchez et al. (2009).

Inicialmente, procedeu-se ao cálculo das estatísticas descritivas dos teores observados de $\mathrm{K}$ disponível (média, desvio-padrão, mínimo, máximo, coeficiente de variação, assimetria e curtose), para identificar tendências, dispersão e forma de distribuição dos dados. $\mathrm{O}$ programa $\mathrm{R}$ foi utilizado para as avaliações das estatísticas descritivas (R Development Core Team, 2010). Além disso, a reprodução dos dados amostrais, 
pelos modelos de simulação e estimação, foi verificada com estatísticas descritivas e variogramas.

A dependência espacial dos teores de K disponível no solo foi caracterizada por meio do variograma, de acordo com a teoria das variáveis regionalizadas (Soares, 2006). Os ajustes dos variogramas experimentais foram feitos com base na menor soma de quadrado dos resíduos (SQR) e no maior coeficiente de determinação $\left(\mathrm{R}^{2}\right)$ (Isaaks \& Srivastava, 1989).

$\mathrm{Na}$ estimativa da KO, a dependência espacial dos teores de $\mathrm{K}$ foi quantificada por meio do variograma. $\mathrm{O}$ teor de $\mathrm{K}$ em um local não amostrado $\left(\mathrm{x}_{0}\right)$ foi estimado com uso do estimador $\hat{z}\left(x_{0}\right)$, definido como:

$$
\mathrm{z}\left(\mathrm{x}_{0}\right)=\sum_{\mathrm{i}=1}^{\mathrm{N}} \lambda_{\mathrm{i}} \mathrm{z}\left(\mathrm{x}_{\mathrm{i}}\right), \operatorname{com} \sum_{\mathrm{i}=1}^{\mathrm{N}} \lambda_{\mathrm{i}}=1,
$$

em que $\hat{z}\left(x_{0}\right)$ é o valor estimado de $\mathrm{K}$ no ponto $0 ; \mathrm{N}$ é o número de valores utilizados na estimação; $\lambda_{\mathrm{i}}$ é o peso associado a cada valor observado; e $\mathrm{z}\left(\mathrm{x}_{\mathrm{i}}\right)$ é o teor observado de K no ponto i (Soares, 2006).

A variância da $\mathrm{KO}\left(\sigma^{2}\right)$, em locais não amostrados $\left(\mathrm{x}_{0}\right)$, foi calculada por:

$$
\sigma^{2}\left(\mathrm{x}_{0}\right)=\sum_{\mathrm{i}=1}^{\mathrm{N}} \lambda_{\mathrm{i}} \gamma\left(\mathrm{x}_{0}, \mathrm{x}_{\mathrm{i}}\right)+\mu,
$$

em que $\gamma\left(\mathrm{x}_{0}, \mathrm{x}_{\mathrm{i}}\right)$ é a semivariância entre o local a ser estimado $\mathrm{x}_{0}$ e o i-ésimo ponto amostrado; e $\mu$ é o parâmetro de Lagrange utilizado para minimizar a variância da KO (Soares, 2006).

No procedimento da SSG, os dados devem ser transformados para que tenham distribuição normal padronizada (com média igual a 0 e variância igual a 1). A normalidade dessa transformação foi verificada por meio do teste de normalidade de Shapiro-Wilk, a $5 \%$ de probabilidade. O procedimento da SSG consiste em refinar a malha amostral e definir um caminho aleatório que permita que cada ponto a ser simulado seja visitado apenas uma vez. Para cada um desses pontos, a função de distribuição acumulada condicional (FDAC) gaussiana é determinada por meio do estimador da krigagem simples, com uso do variograma dos dados normalizados. Posteriormente, um valor normal padronizado é sorteado a partir da FDAC e adicionado ao conjunto de dados condicionais, que consiste nos pontos observados e nos previamente simulados dentro da vizinhança do ponto a ser simulado. Esse procedimento é repetido até que todos os pontos sejam simulados. Por fim, os dados normalizados são transformados para a escala original dos dados obtidos. Para gerar múltiplas realizações, essa sequência deve ser repetida com diferentes caminhos aleatórios, para cada realização (Soares, 2006).

A SSI é baseada na abordagem sequencial, que inclui os dados observados e todos os valores previamente simulados dentro de uma vizinhança, e o estimador da krigagem indicatriz (KI) é usado para modelar as FDACs em cada ponto não amostrado. O procedimento consiste em transformar cada valor observado em um vetor de indicadores ( 0 ou 1$)$, de acordo com os valores de corte $\mathrm{z}_{\mathrm{k}}$, por meio da seguinte função indicadora: $\mathrm{I}\left(\mathrm{x}_{\alpha} ; \mathrm{z}_{\mathrm{l}}\right)=1$, se $\mathrm{z}\left(\mathrm{x}_{\alpha} \leq \mathrm{z}_{\mathrm{l}}\right)$; e $\mathrm{I}\left(\mathrm{x}_{\alpha} ; \mathrm{z}_{\mathrm{l}}\right)=0$, no caso contrário, com $1=1, \ldots, \mathrm{L} \mathrm{e} \alpha=1, \ldots, \mathrm{n}$. Em seguida, os $\mathrm{L}$ variogramas indicadores são calculados e modelados, de acordo com os valores de corte $\mathrm{z}_{\mathrm{l}}$.

No presente trabalho, foram utilizados quatro valores de corte $\left(1,2,1,7,2,2\right.$ e $\left.3,1 \mathrm{mmol}_{\mathrm{c}} \mathrm{dm}^{-3}\right)$ que correspondem, respectivamente, aos percentis 0,2 , $0,4,0,6$ e 0,8 da distribuição dos teores de $\mathrm{K}$ nas amostras. Assim, foram gerados novos conjuntos de dados compostos de valores 0 e 1 , de acordo com a função indicadora. Um caminho aleatório, que passe por todos os pontos a serem simulados, é estabelecido ao se visitar cada ponto apenas uma vez. Dessa forma, para cada ponto a ser simulado ao longo do caminho, a FDAC inicial é determinada com o algoritmo da krigagem indicatriz, e quaisquer desvios de ordem entre os valores estimados (maiores que 1 ou menores que 0) são corrigidos para determinar a FDAC completa. $\mathrm{O}$ valor simulado é obtido a partir da FDAC para cada local e adicionado ao conjunto de variáveis indicadoras condicionantes (valores observados e previamente simulados), para modelar a FDAC da próxima localização ao longo do caminho aleatório (Soares, 2006).

Para obter várias realizações, o processo sequencial é repetido com diferentes caminhos aleatórios. No presente trabalho, foram geradas 200 realizações, para cada método de simulação (SSG e SSI), tendose utilizado o programa WinGslib (Deutsch \& Journel, 1998). Para representar o conjunto de 200 realizações e verificar a reprodução dos valores observados de K, foram sorteadas, aleatoriamente, as realizações 11, 60, 94 e 143.

A partir das realizações geradas com os algoritmos da SSG e da SSI, foram obtidas diferentes medidas de estimativa e de incerteza. A média de todos os 200 mapas simulados, calculada ponto a ponto, 
forneceu uma estimativa denominada E-type (Deutsch \& Journel, 1998). Da mesma forma, o desvio-padrão de todos os mapas simulados, calculado ponto a ponto, forneceu o mapa espacial de desvio, que foi utilizado para avaliar a incerteza local (Bourennane et al., 2007). O mapa de desvio-padrão representa a variação do teor de $K$ nas $n$ realizações simuladas $(n=200)$.

A incerteza espacial foi contabilizada por meio do cálculo da probabilidade conjunta (Pc) (Teixeira et al., 2013; Oliveira et al., 2013). Previamente, calculou-se a probabilidade de os valores estimados pontualmente excederem determinado limite crítico (Delbari et al., 2009; Zhao et al., 2009). O limite crítico adotado foi o valor da mediana dos teores de $\mathrm{K}$ disponíveis. Em seguida, o cálculo da Pc foi efetuado. A Pc auxilia na determinação da confiabilidade em delinear áreas com alta probabilidade de exceder determinado valor crítico e, dessa forma, representa um indicativo da incerteza espacial.

Para o cálculo da Pc, foram consideradas as áreas que apresentaram probabilidade maior ou igual a $95 \%$ de excederem o limite crítico. O procedimento consiste na contabilização do número de realizações em que todos os locais aparecem simultaneamente simulados acima do valor crítico. Para mais detalhes, veja Juang et al. (2004).

Utilizou-se a metodologia proposta por Deutsch (1997) para examinar a acurácia na reprodução da FDAC dos dados amostrais, no conjunto de realizações da SSG e da SSI. Verificaram-se as proporções de valores dos dados do conjunto de validação que pertencem a intervalos de probabilidade (IP) simétricos, calculados a partir da FDAC F(u, z|(n)). Em qualquer localização $\mathrm{u}$, o conhecimento da FDAC permite calcular uma série de IP simétricos limitados pelos quantis da FDAC $(1-p) / 2$ e $(1+p) / 2$. As frações de valores verdadeiros a que pertencem cada IP simétrico foram obtidas com a consideração dos dados de validação $F\left(u_{j}, z \mid(n)\right), j=$ $1, \ldots, \mathrm{N}_{\mathrm{v}}=63$, por meio de:

$$
\begin{gathered}
\bar{\xi}_{(\mathrm{p})}=\frac{1}{\mathrm{~N}} \sum_{\mathrm{j}=1}^{\mathrm{N}_{\mathrm{v}}} \xi\left(\mathrm{u}_{\mathrm{j}}, \mathrm{p}\right) \quad \forall \pi \in[0,1], \\
\xi\left(\mathrm{u}_{\mathrm{j}}, \mathrm{p}\right)= \begin{cases}1 & \operatorname{seF}^{-1}\left(\mathrm{u}_{\mathrm{j}},(1-\mathrm{p}) / 2\right)<\mathrm{z}\left(\mathrm{u}_{\mathrm{j}}\right) \leq \widehat{\mathrm{F}}^{-1}\left(\mathrm{u}_{\mathrm{j}},(1+\mathrm{p}) / 2\right) \\
0 & \text { caso contrário. }\end{cases}
\end{gathered}
$$

$\mathrm{O}$ algoritmo de simulação usado para gerar as FDACs é considerado acurado quando $\bar{\xi}(\mathrm{p}) \geq \mathrm{p}, \forall \mathrm{p}$. O gráfico de dispersão, denominado gráfico de acurácia, foi gerado a partir das frações calculadas vs. o conjunto de probabilidades p. A estatística $G$ foi utilizada para mensurar, no gráfico de acurácia, o afastamento dos pontos em relação à linha de $45^{\circ}$, e foi numericamente calculada pela equação (Deutsch, 1997):

$$
\mathrm{G}=1-\int_{0}^{1}[3 a(p)-2][\bar{\xi}(\mathrm{p})-\mathrm{p}] \mathrm{dp},
$$

em que a(p) é igual a 1 , se $\bar{\xi}(\mathrm{p}) \geq \mathrm{p}$, e igual a 0 , caso contrário. Considerou-se que valores de $\mathrm{G}$ iguais à unidade indicam máxima eficiência no ajuste do modelo, ou seja, $\bar{\xi}(p)=p, \forall p \in[0,1]$.

Os valores dos dados de validação e os estimados, fornecidos pelos métodos de simulação, foram utilizados para o cálculo da raiz do erro quadrático médio (REQM) (Bourennane et al., 2007; Teixeira et al., 2012), de acordo com a seguinte a equação:

$$
\operatorname{REQM}=\left\{\frac{1}{\mathrm{n}} \sum_{\mathrm{i}=1}^{\mathrm{n}}\left[\mathrm{z}\left(\mathrm{x}_{\mathrm{i}}\right)-\overline{\mathrm{z}}\left(\mathrm{x}_{\mathrm{i}}\right)\right]^{2}\right\}^{0,5},
$$

em que n é o número de valores utilizados na validação, no presente trabalho $\mathrm{n}=63 ; \mathrm{z}\left(\mathrm{x}_{\mathrm{i}}\right)$ é o valor da propriedade no ponto i; e $\hat{z}\left(x_{\mathrm{i}}\right)$ é o valor estimado da propriedade no ponto i. Menores valores de REQM estão relacionados à maior acurácia nas predições (Chai et al., 2007).

\section{Resultados e Discussão}

As estatísticas descritivas dos dados de validação foram próximas às dos dados amostrais (Tabela 1), e não houve diferença significativa entre os dois conjuntos de dados pelo teste $\mathrm{t}$ de Student, a 5\% de probabilidade. A média dos teores de $\mathrm{K}$ disponível observados $\left(2,99 \mathrm{mmol}_{\mathrm{c}} \mathrm{dm}^{-3}\right)$ indica a presença de valores elevados, para a cultura da cana-de-açúcar (Raij et al., 1997). Marques Júnior et al. (2008) e Souza et al. (2010) relataram, respectivamente, teores médios e elevados de $\mathrm{K}$ em solos sob o cultivo da espécie. Embora a média dos teores de $\mathrm{K}$ esteja bem próxima à faixa de alta fertilidade $\left(3,1-6,0 \mathrm{mmol}_{\mathrm{c}} \mathrm{dm}^{-3}\right)$, os valores apresentaram grande amplitude $\left(0,30-11,90 \mathrm{mmol}_{\mathrm{c}}\right.$ $\left.\mathrm{dm}^{-3}\right)$. O coeficiente de variação (CV) de $66,96 \%$, para os dados amostrais, indica alta variabilidade, de acordo com a classificação de Warrick \& Nielsen (1980), em que valores de $\mathrm{CV}>35 \%$ revelam heterogeneidade dos 
dados e média pouco significativa. Portanto, a aplicação do fertilizante potássico, ao se levar em consideração apenas o valor médio da propriedade, pode sub ou superdimensionar as doses a serem aplicadas.

As estatísticas referentes às quatro realizações individuais da SSG e da SSI (Tabela 1) mantiveram os mesmos valores mínimos e máximos dos dados amostrais. Nas realizações da SSG, os valores do $\mathrm{CV}$ e do desvio-padrão foram mais próximos aos dos dados amostrais do que nas realizações da SSI. No procedimento da $\mathrm{KO}$, o $\mathrm{CV}$ foi aproximadamente $44 \%$ menor que o dos dados amostrais, o que revela o efeito da minimização da variância. $\mathrm{O}$ estimador $\mathrm{KO}$ também apresentou valor mínimo superestimado e valor máximo subestimado, em comparação aos dados originais, o que confirma o efeito de suavização típico desse estimador. Esse cenário também foi encontrado por Delbari et al. (2010), ao analisar as técnicas KO, SSG e SSI, para a modelagem da variabilidade espacial do carbono orgânico do solo.

A média, o desvio-padrão e o $\mathrm{CV}$ da E-type de ambos os métodos de simulação apresentaram valores próximos aos da $\mathrm{KO}$, além de padrões espaciais semelhantes aos do mapa da KO. Segundo Chilès \& Delfiner (1999), a semelhança entre os padrões espaciais dos mapas estimados pela $\mathrm{KO}$ e as realizações E-type indicam que o procedimento de simulação foi realizado com número adequado de realizações. A utilização de um número adequado de realizações garante a avaliação do espaço de incertezas das estimativas em estudo (Goovaerts, 1999), uma vez que o conjunto de realizações forneceria um campo probabilístico grande o suficiente para possibilitar a seleção de realizações aleatórias que representassem o conjunto simulado.

A caracterização da estrutura da variabilidade espacial foi determinada com o ajuste de modelos teóricos aos variogramas experimentais. Para a $\mathrm{KO}$, utilizou-se a transformação logarítmica dos dados para a correção da assimetria da distribuição (Tabela 1). A transformação logarítmica é recomendada para conjuntos de valores com assimetria positiva maior que a unidade (Webster \& Oliver, 2009). Kerry \& Oliver (2007) afirmam que o efeito da assimetria é maior quando os valores de assimetria são maiores que a unidade, principalmente quando associados a pequenos números de amostras. Assim, a transformação dos dados objetivou a correção da forma do variograma, o que aumentou a dependência espacial e reduziu o efeito pepita o que, consequentemente, melhorou a precisão e a acurácia das estimativas.

Tabela 1. Estatísticas descritivas dos teores de potássio no solo.

\begin{tabular}{|c|c|c|c|c|c|c|c|c|}
\hline Parâmetro & $\mathrm{N}$ & $\begin{array}{c}\text { Média } \\
\left(\mathrm{mmol}_{\mathrm{c}} \mathrm{dm}^{-3}\right)\end{array}$ & $\mathrm{DP}$ & $\begin{array}{l}\mathrm{CV} \\
(\%)\end{array}$ & Mínimo & Máximo & Assimetria & Curtose \\
\hline Dados amostrais $^{(1)}$ & 563 & 2,99 & 1,54 & 66,96 & 0,30 & 11,90 & 2,38 & 8,18 \\
\hline Dados de validação(2) & 63 & 2,62 & 1,84 & 70,37 & 0,50 & 8,00 & 1,29 & 0,95 \\
\hline \multirow[t]{2}{*}{ Krigagem ordinária } & 8.640 & 2,18 & 0,64 & 29,66 & 1,00 & 5,55 & 1,25 & 2,63 \\
\hline & \multicolumn{8}{|c|}{ Simulação sequencial gaussiana } \\
\hline E-type & 8.640 & 2,20 & 0,62 & 28,22 & 0,30 & 11,90 & 3,03 & 27,84 \\
\hline Realização 11 & 8.640 & 2,26 & 1,48 & 65,46 & 0,30 & 11,90 & 2,34 & 8,07 \\
\hline Realização 60 & 8.640 & 2,19 & 1,38 & 63,34 & 0,30 & 11,90 & 2,37 & 8,66 \\
\hline Realização 94 & 8.640 & 2,22 & 1,41 & 63,44 & 0,30 & 11,90 & 2,35 & 8,27 \\
\hline \multirow[t]{2}{*}{ Realização 143} & 8.640 & 2,17 & 1,39 & 64,01 & 0,30 & 11,90 & 2,40 & 8,80 \\
\hline & \multicolumn{8}{|c|}{ Simulação sequencial indicatriz } \\
\hline E-type & 8.640 & 2,76 & 0,71 & 25,85 & 0,30 & 11,90 & 1,67 & 11,80 \\
\hline Realização 11 & 8.640 & 2,82 & 2,50 & 92,06 & 0,30 & 11,90 & 1,92 & 2,86 \\
\hline Realização 60 & 8.640 & 2,73 & 2,52 & 92,24 & 0,30 & 11,90 & 2,09 & 3,44 \\
\hline Realização 94 & 8.640 & 2,72 & 2,54 & 93,20 & 0,30 & 11,90 & 2,04 & 3,38 \\
\hline Realização 143 & 8.640 & 2,69 & 2,47 & 91,73 & 0,30 & 11,90 & 2,05 & 3,55 \\
\hline
\end{tabular}

(1)Dados utilizados nos procedimentos de simulações e estimativas por krigagem ordinária. ${ }^{(2)}$ Dados utilizados na validação externa. $\mathrm{N}$, número de observações; DP, desvio-padrão; CV, coeficiente de variação. 
A transformação normal dos dados originais dos teores de $\mathrm{K}$ foi realizada para atender a pré-requisito da SSG, o qual demanda que os dados avaliados devem apresentar distribuição normal ou muito próxima a ela.

O modelo esférico foi o que apresentou os melhores ajustes ao variograma dos dados normalizados. De acordo com a classificação de Cambardella et al. (1994), o grau de dependência espacial - GDE $\left(\mathrm{C}_{0} /\left(\mathrm{C}_{0}+\mathrm{C}_{1}\right)\right)$ - foi moderado, tanto para os dados com transformação logarítmica quanto para aqueles com transformação normal. O modelo esférico foi o que apresentou os melhores ajustes aos quatro variogramas indicadores. Os variogramas dos percentis 0,20 e 0,40 apresentaram GDE fraco, enquanto os dos percentis 0,60 e 0,80 apresentaram GDE moderado.

As realizações individuais da SSG apresentaram menor variabilidade em pequena escala do que as realizações da SSI (Figura 1). Zhao et al. (2005) relataram realizações da SSI com semelhantes padrões de variabilidade para valores extremos de carbono orgânico. Os padrões espaciais das simulações individuais geradas por ambos os métodos apresentam maior variabilidade com teores altos e intermediários de $\mathrm{K}$.

O histograma e o variograma dos dados amostrais não foram reproduzidos pelos gerados pela $\mathrm{KO}$ (Figura 2). O estimador KO subestimou a variabilidade em pequena escala, representada no variograma com menor efeito pepita. Esse mesmo cenário foi constatado por Delbari et al. (2010) e está fortemente relacionado à característica de suavização do método da $\mathrm{KO}$. As realizações individuais da SSG reproduziram melhor o variograma e o histograma dos dados amostrais que a $\mathrm{KO}$, resultado similar ao observado por Bourennane et al. (2010). Já a SSI reproduz apenas os modelos dos variogramas indicadores. A reprodução dos variogramas correspondentes aos quantis 0,20 , $0,40,0,60$ e 0,80 da distribuição dos teores de $\mathrm{K}$ nas realizações está descrita na Figura 3. A SSI reproduziu melhor a continuidade espacial em médias e grandes escalas, enquanto os variogramas das realizações sorteadas da SSG reproduziram melhor a variabilidade espacial em pequenas escalas.

Os mapas E-type, gerados a partir da SSI e da SSG (Figura 4), foram semelhantes ao mapa gerado a partir da KO. No entanto, as estimativas E-type das simulações preservaram a variação (mínimo e máximo) dos dados amostrais (Tabela 1). Outros trabalhos com KO e SSI (Juang et al., 2004; Zhao et al., 2009), bem como com KO e SSG (Bourennane et al., 2007; Goovaerts et al., 2008), apresentaram resultados semelhantes. Essa característica dos mapas E-type indica grande potencial prático para a definição de zonas homogêneas, para manejo específico. A identificação dessas zonas por meio das realizações individuais da SSG pode dificultar o entendimento do usuário e a aplicação da técnica, uma vez que inúmeros mapas igualmente prováveis

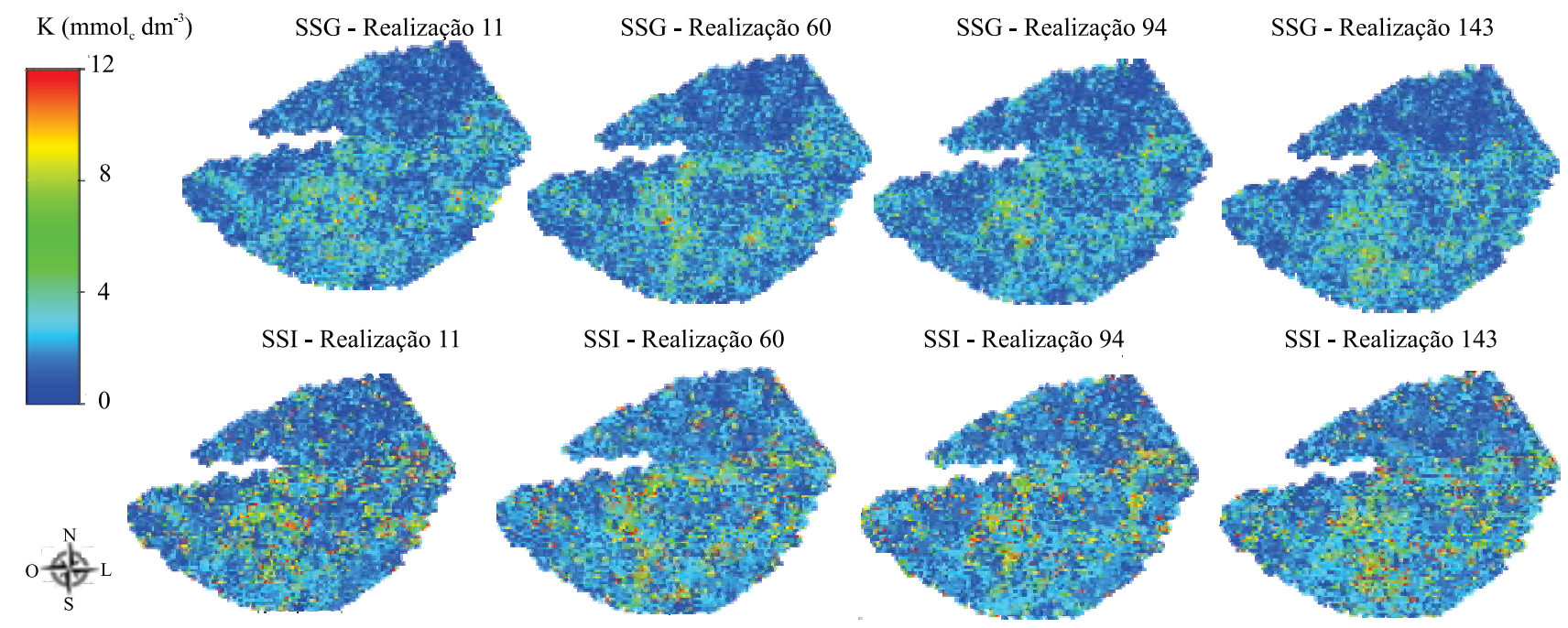

Figura 1. Mapas dos teores estimados de $\mathrm{K}$ nas realizações sorteadas 11, 60, 94 e 143, para as simulações sequencial gaussiana (SSG) e sequencial indicatriz (SSI). 
são produzidos, ao invés de um único mapa com variância mínima (Soares, 2006). Outro fator que pode inviabilizar a utilização das realizações é o alto grau de detalhamento reproduzido pela SSG, já que as máquinas e os implementos disponíveis para aplicações em taxas variáveis não contemplam essa alta erraticidade dos padrões estimados por essa simulação.

Os mapas de desvios gerados por ambos os algoritmos de simulação mostram alta incerteza espacial na parte sudoeste da área de estudo (Figura 5), local em que os maiores teores de $\mathrm{K}$ foram obtidos. Entretanto, no mapa
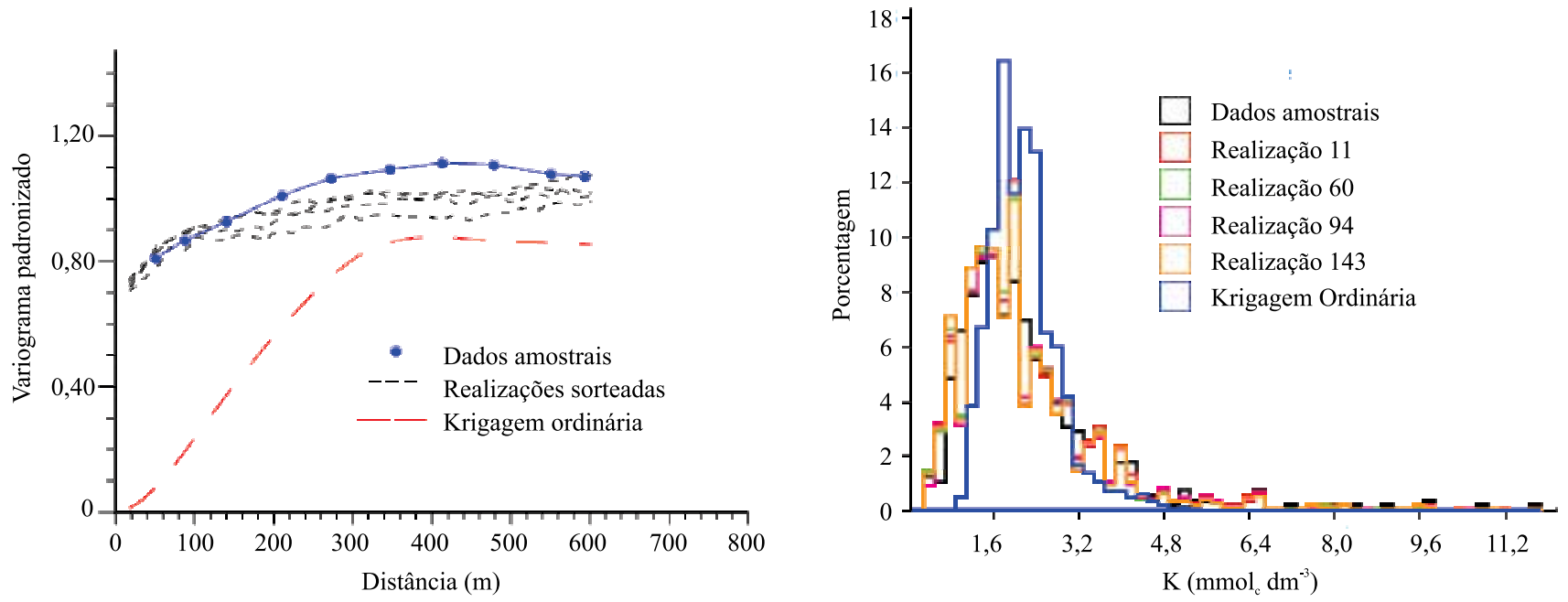

Figura 2. Variogramas padronizados e histogramas dos teores de $\mathrm{K}$ para os dados amostrais, nas realizações sorteadas da simulação sequencial gaussiana (SSG) 11, 60, 94 e 143, e na krigagem ordinária.

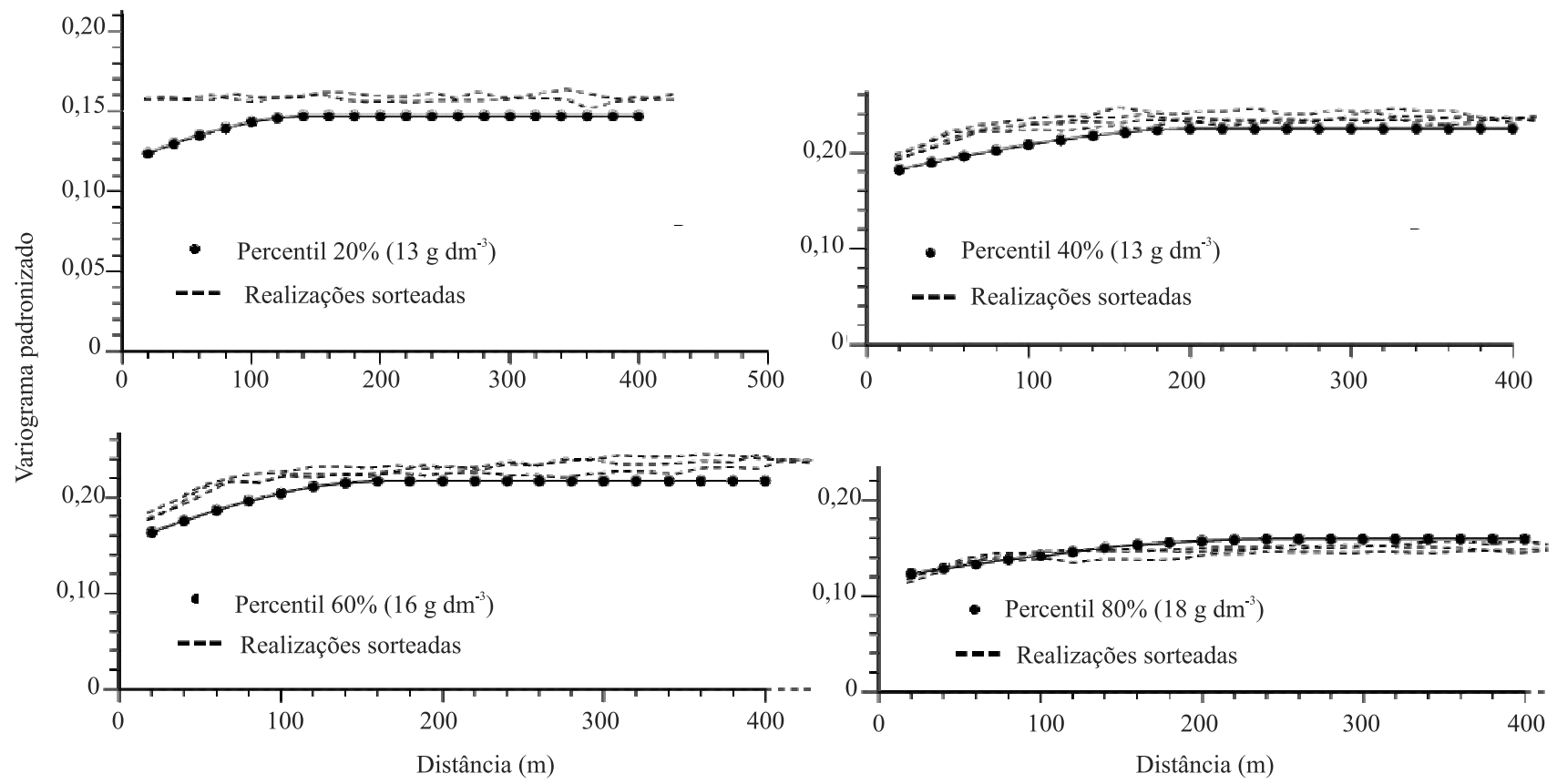

Figura 3. Variogramas indicadores padronizados dos teores de K, para os valores de corte e as realizações sorteadas da simulação sequencial indicatriz 11, 60, 94 e 143. 
de desvios da SSI, as áreas com alta incerteza foram maiores que aquelas indicadas pela SSG. A identificação e a delimitação dessas localidades favorecem $o$ planejamento amostral da área, e essas áreas devem ser amostradas mais detalhadamente para diminuição das incertezas e, consequentemente, para melhor captação da real variabilidade do nutriente no solo.

Os desvios-padrão locais associados às estimativas da KO apresentaram menor variação, ao longo da área de estudo (Figura 5). Esse resultado se deve ao fato de o desvio-padrão da KO ser independente do valor real da amostra. Essa relação foi confirmada por meio da representação gráfica do diagrama de dispersão da E-type vs. desvio-padrão (Figura 6). Bourennane et al. (2007) e Delbari et al. (2009) obtiveram resultado similar para estimativas do conteúdo de água do solo.

Nos algoritmos de simulação, os valores médios de $\mathrm{K}$ apresentaram evidente relação com os desvios- padrão locais (Figura 6). Assim, valores pequenos a intermediários das médias locais de $\mathrm{K}\left(<3 \mathrm{mmol}_{\mathrm{c}}\right.$ $\left.\mathrm{dm}^{-3}\right)$ apresentam baixa incerteza local $\left(<1,5 \mathrm{mmol}_{\mathrm{c}}\right.$ $\left.\mathrm{dm}^{-3}\right)$. Esses resultados destacam a necessidade da avaliação precisa da incerteza associada aos métodos de interpolação. Essas informações devem ser incorporadas aos processos de tomada de decisão, o que permitiria o estabelecimento preciso de áreas para fins de manejo específico.

A análise visual e comparativa dos mapas das realizações selecionadas (Figura 1) é o primeiro indicativo da incerteza espacial do atributo avaliado (Teixeira et al., 2013). No entanto, apenas com o cálculo da Pc já é possível quantificar essa incerteza (Juang et al., 2004). No presente trabalho, o limite de corte adotado foi o valor da mediana dos teores de $\mathrm{K}$ $\left(1,9 \mathrm{mmol}_{\mathrm{c}} \mathrm{dm}^{-3}\right)$ e, em ambas as simulações, $3,5 \% \mathrm{da}$ área total apresentaram probabilidade superior a $95 \%$ de excederem o valor de $1,9 \mathrm{mmol}_{\mathrm{c}} \mathrm{dm}^{-3}$. Contudo, em

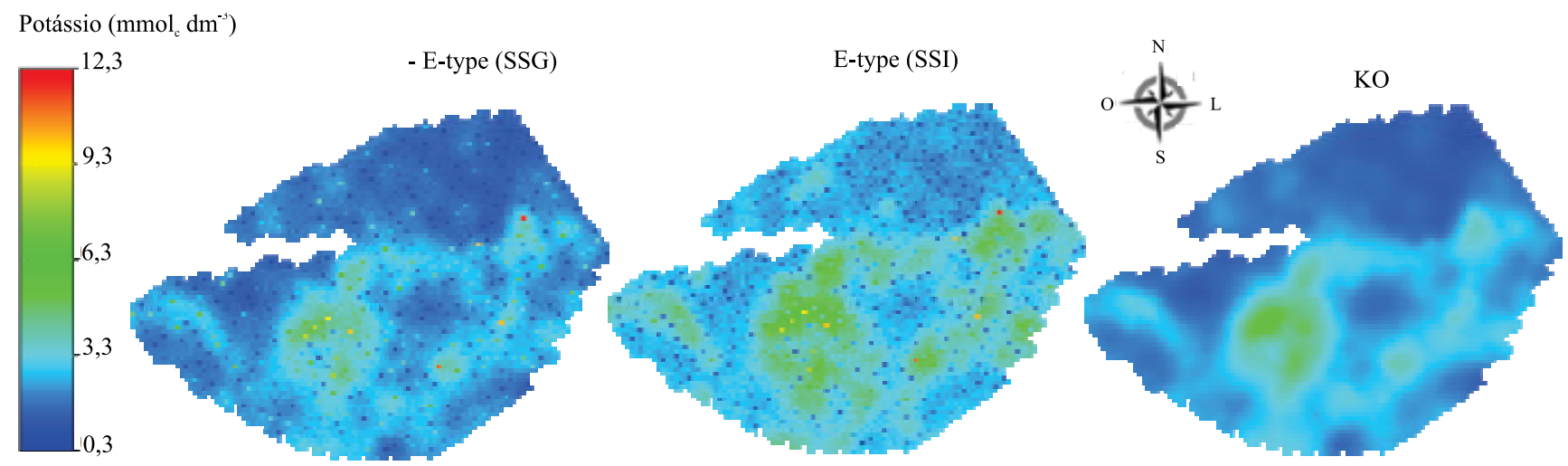

Figura 4. Estimativa E-type das simulações sequencial gaussiana (SSG) e sequencial indicatriz (SSI), e da krigagem ordinária (KO) dos teores de $\mathrm{K}$.
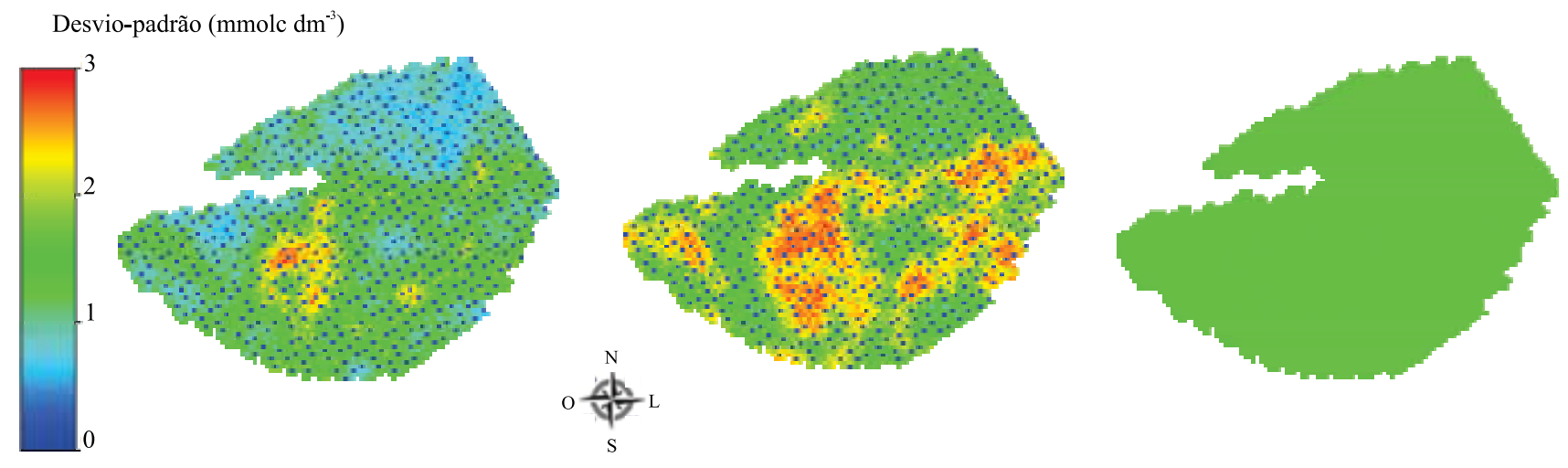

Figura 5. Mapas do desvio-padrão dos teores de K obtidos por: A, simulação sequencial gaussiana; B, simulação sequencial indicatriz; e krigagem ordinária. 
apenas $66,7 \%$ (SSI) e $60,3 \%$ (SSG) das 200 realizações individuais, a totalidade dos pontos pertencentes àquela área foi simulada conjuntamente com valores acima do limite crítico adotado. Assim, de acordo com os valores da Pc, pode-se afirmar que as estimativas realizadas pela SSI apresentam menor incerteza espacial do que as realizadas pela SSG.

A análise visual da Figura 7 mostra que, para o algoritmo da $\mathrm{SSG}$, os desvios dos pontos em relação à reta 1:1 foram maiores do que para o algoritmo da SSI. A SSG apresentou menor valor de $G(0,68)$ do que o calculado a partir da SSI $(0,88)$, indício de que o desempenho por parte da SSI foi $22 \%$ mais acurado, na modelagem da FDAC dos teores de K. Delbari et al. (2010) relataram resultados semelhantes.

O valor do REQM, calculado a partir dos dados amostrais (63 pontos) e das 200 realizações obtidas de cada método de simulação (SSG e SSI), foi maior para a SSG $(2,45)$ do que para a SSI $(1,58)$, o que evidencia que a modelagem dos teores de $\mathrm{K}$ por meio da SSI foi $35 \%$ mais acurada do que a por meio da SSG.
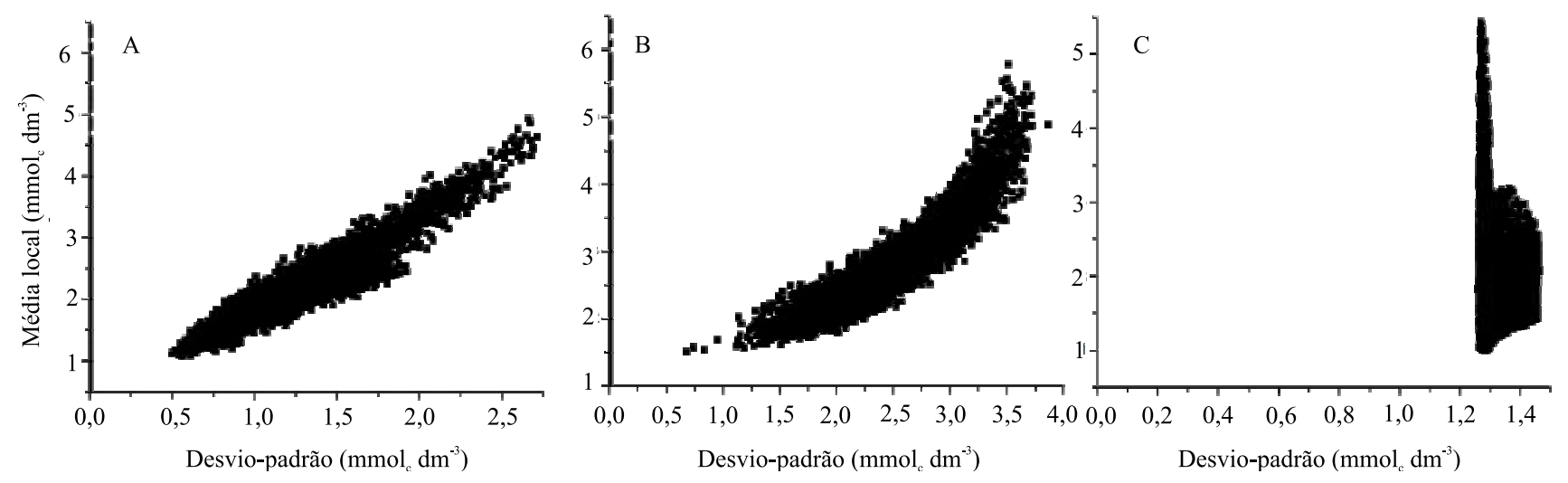

Figura 6. Gráfico de dispersão do desvio-padrão vs. média local dos teores de K obtidos por meio de: A, simulação sequencial gaussiana; B, simulação sequencial indicatriz; e C, krigagem ordinária.
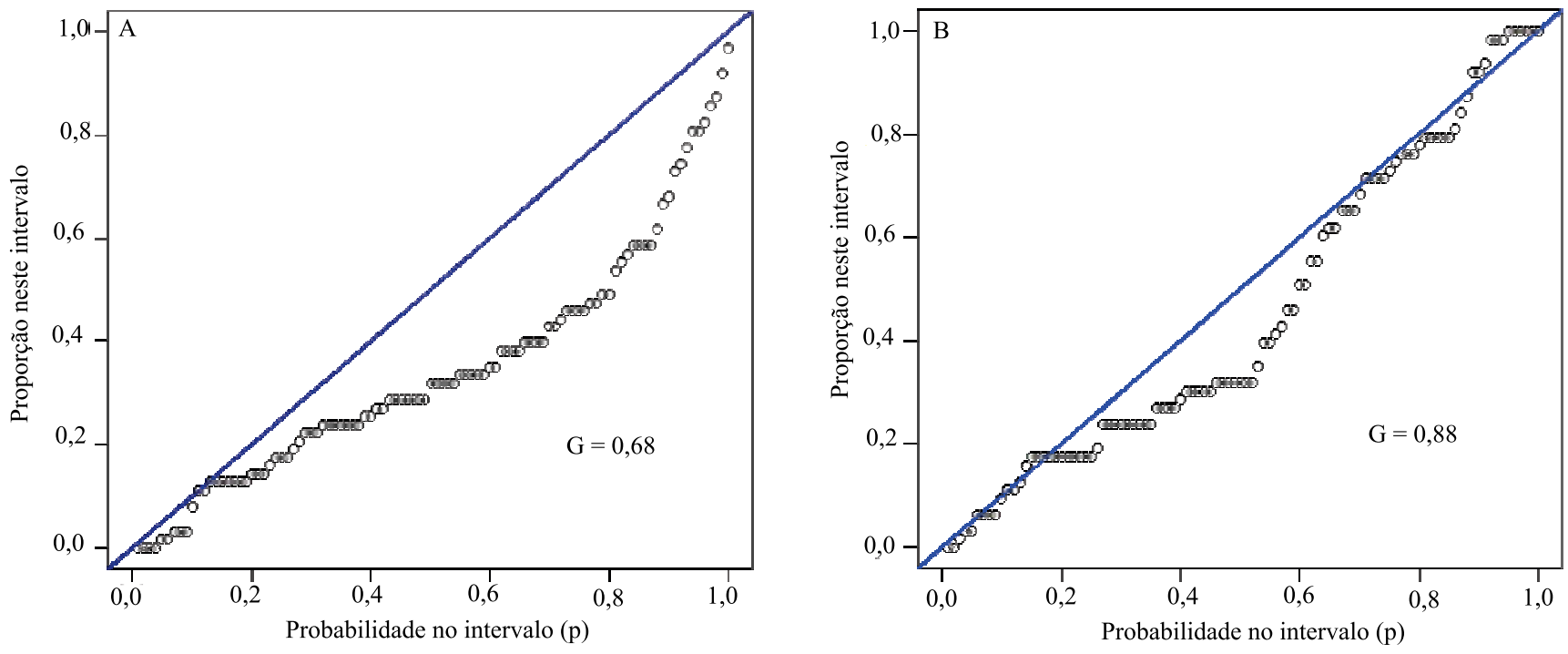

Figura 7. Gráfico de acurácia e estatística G para: A, simulação sequencial gaussiana; e B, simulação sequencial indicatriz dos teores de K. 


\section{Conclusões}

1. A simulação sequencial gaussiana ( $\mathrm{SSG}$ ) e a simulação sequencial indicatriz (SSI) apresentam maior eficiência do que a krigagem ordinária, na modelagem da incerteza da distribuição espacial dos teores de $\mathrm{K}$ disponível em área cultivada com cana-de-açúcar.

2. A SSI apresenta melhor desempenho que a SSG, na estimativa dos teores de $\mathrm{K}$ disponível.

\section{Agradecimentos}

À Coordenação de Aperfeiçoamento de Pessoal de Nível Superior (Capes), pelo apoio financeiro e pela concessão de bolsas.

\section{Referências}

BENITES, V. de M.; CARVALHO, M. da C.S.; RESENDE, A.V.; POLIDORO, J.C.; BERNARDI, A.C.C.; OLIVEIRA, F.A. de. Potássio, cálcio e magnésio. In: SIMPÓSIO SOBRE BOAS PRÁTICAS PARA USO EFICIENTE DE FERTILIZANTES, 2009, Piracicaba. Boas práticas para uso eficiente de fertilizantes: anais. Piracicaba: IPNI, 2010. v.2, p.134-203.

BOURENNANE, H.; DOUAY, F.; STERCKEMAN, T.; VILLANNEAU, E.; CIESIELSKI, H.; KING, D.; BAIZE, D. Mapping of anthropogenic trace elements inputs in agricultural topsoil from Northern France using enrichment factors. Geoderma, v.157, p.165-174, 2010. DOI: 10.1016/j.geoderma.2010.04.009.

BOURENNANE, H.; KING, D.; COUTURIER, A.; NICOULLAUD, B.; MARY, B.; RICHARD, G. Uncertainty assessment of soil water content spatial patterns using geostatistical simulations: an empirical comparison of a simulation accounting for single attribute and a simulation accounting for secondary information. Ecological Modelling, v.205, p.323-335, 2007. DOI: 10.1016/j.ecolmodel.2007.02.034.

CAMARGO, L.A.; MARQUES JÚNIOR, J.; PEREIRA, G.T.; ALLEONI, L.R.F. Spatial correlation between the composition of the clay fraction and contents of available phosphorus of an Oxisol at hillslope scale. Catena, v.100, p.100-106, 2013. DOI: 10.1016/j. catena.2012.07.016.

CAMBARDELLA, C.A.; MOORMAN, T.B.; NOVAK, J.M.; PARKIN, T.B.; KARLEN, D.L.; TURCO, R.F.; KONOPKA, A.E. Field-scale variability of soil properties in central Iowa soils. Soil Science Society of America Journal, v.58, p.1501-1511, 1994. DOI: 10.2136/sssaj1994.03615995005800050033x.

CHAI, X.; HUANG, Y.; YUAN, X. Accuracy and uncertainty of spatial patterns of soil organic matter. New Zealand Journal of Agricultural Research, v.50, p.1141-1148, 2007. DOI: 10.1080/00288230709510396.

CHILÈS, J.-P.; DELFINER, P. Geostatistics: modeling spatial uncertainty. New York: Wiley, 1999. 695p. DOI: 10.1002/9780470316993.
COMPANHIA NACIONAL DE ABASTECIMENTO. Acompanhamento de safra brasileira - cana-de-açúcar - safra 2013/14 - quarto levantamento - abril/2014. Disponível em: <http://www.conab.gov.br/OlalaCMS/uploads/ arquivos/14_04_10_09_00_57_boletim_cana_portugues_-_4o_ lev_-_13.pdf $>$. Acesso em: 4 ago. 2014.

DELBARI, M.; AFRASIAB, P.; LOISKANDL, W. Using sequential Gaussian simulation to assess the field-scale spatial uncertainty of soil water content. Catena, v.79, p.163-169, 2009. DOI: 10.1016/j.catena.2009.08.001.

DELBARI, M.; LOISKANDL, W.; AFRASIAB, P. Uncertainty assessment of soil organic carbon content spatial distribution using geostatistical stochastic simulation. Australian Journal of Soil Research, v.48, p.27-35, 2010. DOI: 10.1071/SR09026.

DEUTSCH, C. Direct assessment of local accuracy and precision. In: BAAFI, E.Y.; SCHOFIELD, N.A. (Ed.). Geostatistics Wollongong '96. Dordrecht: Kluwer Academic, 1997. p.115-125.

DEUTSCH, C.V.; JOURNEL, A.G. GSLIB: geostatistical software library and user's guide. New York: Oxford University, 1998. 369p.

GOOVAERTS, P. Geostatistics in soil science: state-of-the-art and perspectives. Geoderma, v.89, p.1-45, 1999. DOI: 10.1016/ S0016-7061(98)00078-0.

GOOVAERTS, P.; TRINH, H.T.; DEMOND, A.H.; TOWEY, T.; CHANG, S.-C.; GWINN, D.; HONG, B.; FRANZBLAU, A.; GARABRANT, D.; GILLESPIE, B.W.; LEPKOWSKI, J.; ADRIAENS, P. Geostatistical modeling of the spatial distribution of soil dioxin in the vicinity of an incinerator. 2. Verification and calibration study. Environmental Science and Technology, v.42, p.3655-3661, 2008. DOI: 10.1021/es7024966.

ISAAKS, E.H.; SRIVASTAVA, R.M. An introduction to applied geostatistics. Oxford: University Press, 1989. 592p.

JUANG, K.-W.; CHEN, Y.-S.; LEE, D.-Y. Using sequential indicator simulation to assess the uncertainty of delineating heavymetal contaminated soils. Environmental Pollution, v.127, p.229238, 2004. DOI: 10.1016/j.envpol.2003.07.001.

KERRY, R.; OLIVER, M.A. Determining the effect of asymmetric data on the variogram. I. Underlying asymmetry. Computers and Geosciences, v.33, p.1212-1232, 2007. DOI: 10.1016/j. cageo.2007.05.008.

MARQUES JÚNIOR, J.; SOUZA, Z.M. de; PEREIRA, G.T.T.; BARBIERI, D.M. Variabilidade espacial de matéria orgânica, P, $\mathrm{K}$ e CTC de um latossolo cultivado com cana-de-açúcar por longo período. Revista de Biologia e Ciências da Terra, v.8, p.143-152, 2008.

OLIVEIRA, I.R. de; TEIXEIRA, D. De B.; PANOSSO, A.R.; CAMARGO, L.A.; MARQUES JÚNIOR, J.; PEREIRA, G.T. Modelagem geoestatística das incertezas da distribuição espacial do fósforo disponível no solo, em área de cana-de-açúcar. Revista Brasileira de Ciência do Solo, v.37, p.1481-1491, 2013. DOI: 10.1590/S0100-06832013000600005.

OTTO, R.; VITTI, G.C.; LUZ, P.H. de C. Manejo da adubação potássica na cultura da cana-de-açúcar. Revista Brasileira de Ciência do Solo, v.34, p.1137-1145, 2010. DOI: 10.1590/S010006832010000400013. 
R DEVELOPMENT CORE TEAM. R: a language and environment for statistical computing. Vienna: R Foundation for Statistical Computing, 2012.

RAIJ, B. van; ANDRADE, J.C. de; CANTARELLA, H.; QUAGGIO, J.A. (Ed.). Análise química para avaliação da fertilidade de solos tropicais. Campinas: Instituto Agronômico, 2001. 285p.

RAIJ, B. van; CANTARELLA, H.; QUAGGIO, J.A; FURLANI, A.M.C. (Ed.). Recomendações de adubação e calagem para o Estado de São Paulo. 2.ed. Campinas: IAC, 1997. 285p.

SANCHEZ, R.B.; MARQUES JÚNIOR, J.; SOUZA, Z.M. de; PEREIRA, G.T.; MARTINS FILHO, M.V. Variabilidade espacial de atributos do solo e de fatores de erosão em diferentes pedoformas. Bragantia, v.68, p.1095-1103, 2009. DOI: 10.1590/ S0006-87052009000400030.

SANTOS, H.G. dos; JACOMINE, P.K.T.; ANJOS, L.H.C. dos; OLIVEIRA, V.A. de; LUMBRERAS, J.F.; COELHO, M.R.; ALMEIDA, J.A. de; CUNHA, T.J.F.; OLIVEIRA, J.B. de. Sistema brasileiro de classificação de solos. 3.ed. rev. e ampl. Brasília: Embrapa, 2013. 353p.

SILVA JUNIOR, J.F. da; MARQUES JÚNIOR, J.; CAMARGO, L.A.; TEIXEIRA, D. De B.; PANOSSO, A.R.; PEREIRA, G.T. Simulação geoestatística na caracterização espacial de óxidos de ferro em diferentes pedoformas. Revista Brasileira de Ciência do Solo, v.36, p.1395-1406, 2012. DOI: 10.1590/S010006832012000600003.

SOARES, A. Geoestatística para ciências da terra e do ambiente. 2.ed. Lisboa: Instituto Superior Técnico, 2006. 214p.

SOUZA, Z.M. de; CERRI, D.G.P.; COLET, M.J.; RODRIGUES, L.H.A.; MAGALHÃES, P.S.G.; MANDONI, R.J.A. Análise dos atributos do solo e da produtividade da cultura de cana-de-açúcar com o uso da geoestatística e árvore de decisão. Ciência Rural, v.40, p.840-847, 2010. DOI: 10.1590/S0103-84782010005000048.
SUN, X.-L.; WU, S.-C.; WANG, H.-L.; ZHAO, Y.; ZHAO, Y.; ZHANG, G.; MAN, Y.B.; WONG, M.H. Uncertainty analysis for the evaluation of agricultural soil quality based on digital soil maps. Soil Science Society of America Journal, v.76, p.13791389, 2012. DOI: 10.2136/sssaj2011.0426.

TEIXEIRA, D. De B.; BICALHO, E. da S.; PANOSSO, A.R.; PERILLO, L.I.; IAMAGUTI, J.L.; PEREIRA, G.T.; LA SCALA, $\mathrm{N}$. Uncertainties in the prediction of spatial variability of soil $\mathrm{CO}_{2}$ emissions and related properties. Revista Brasileira de Ciência do Solo, v.36, p.1466-1475, 2012. DOI: 10.1590/S010006832012000500010.

TEIXEIRA, D.B.; BICALHO, E.S.; CERRI, C.E.P.; PANOSSO, A.R.; PEREIRA, G.T.; LA SCALA, N. Quantification of uncertainties associated with space-time estimates of short-term soil $\mathrm{CO}_{2}$ emissions in a sugar cane area. Agriculture, Ecosystems and Environment, v.167, p.33-37, 2013. DOI: $10.1016 / \mathrm{j}$. agee.2013.01.004.

WARRICK, A.W.; NIELSEN, D.R. Spatial variability of soil physical properties in the field. In: HILLEL, D. (Ed.). Applications of soil physics. New York: Academic, 1980. p.319-344. DOI: 10.1016/B978-0-12-348580-9.50018-3.

WEBSTER, R.; OLIVER, M. A. Geostatistics for environmental scientists. $2^{\text {nd }}$ ed. Oxford: Oxford University, 2009. 315p.

ZHAO, Y.; SHI, X.; YU, D.; WANG, H.; SUN, W. Uncertainty assessment of spatial patterns of soil organic carbon density using sequential indicator simulation, a case study of Hebei province, China. Chemosphere, v.59, p.1527-1535, 2005. DOI: 10.1016/j. chemosphere.2005.01.002.

ZHAO, Y.; XU, X.; DARILEK, J.L.; HUANG, B.; SUN, W.; SHI, $\mathrm{X}$. Spatial variability assessment of soil nutrients in an intense agricultural area, a case study of Rugao County in Yangtze River Delta Region, China. Environmental Geology, v.57, p.1089-1102, 2009. DOI: $10.1007 / \mathrm{s} 00254-008-1399-5$.

Recebido em 3 de dezembro de 2014 e aprovado em 25 de agosto de 2014 